\title{
Potential value of sonazoid-enhanced intraoperative laparoscopic ultrasonography for liver assessment during laparoscopy-assisted colectomy
}

\author{
Tetsuya Itabashi $\cdot$ Akira Sasaki $\cdot$ Koki Otsuka • \\ Toshimoto Kimura • Hiroyuki Nitta • \\ Go Wakabayashi
}

Received: 18 April 2012/ Accepted: 4 March 2013/Published online: 14 May 2013

(C) The Author(s) 2013. This article is published with open access at Springerlink.com

\begin{abstract}
Purpose Laparoscopy-assisted colectomy (LAC) has gained acceptance for the treatment of colorectal cancer. However, conventional palpation of the liver and adequate observation of the abdominal cavity are not achievable during LAC. The aim of this study was to assess the clinical value of using Sonazoid (contrast enhanced)intraoperative laparoscopic ultrasonography (S-IOLUS) in patients with primary colorectal cancer.

Methods From May 2005 to August 2008, 454 patients underwent 339 LACs and 115 open colectomies for colorectal cancer. One hundred forty-eight patients with clinical stage II or III colorectal cancer, as determined by preoperative imaging, who were undergoing LACs were prospectively enrolled.

Results Although IOLUS did not detect any lesions, small hypoechoic lesions were detected by the S-IOLUS $(n=71)$ in the Kupffer-phase view of two patients $(2.8 \%)$. None of the 71 patients who underwent S-IOLUS showed liver metastases within 6 months after LAC. In the conventional IOLUS group $(n=77)$, metastatic lesions were identified in two patients $(2.6 \%)$. The new liver metastases in these two patients were detected within 6 months after LAC.

Conclusions S-IOLUS of the liver during colorectal cancer surgery is useful for staging and as a diagnostic modality. It can identify lesions that are undetectable by preoperative imaging, and may be considered for routine use during LAC.
\end{abstract}

T. Itabashi $(\bowtie) \cdot$ A. Sasaki · K. Otsuka · T. Kimura · H. Nitta · G. Wakabayashi

Department of Surgery, Iwate Medical University School of Medicine, 19-1 Uchimaru, Morioka 020-8505, Japan e-mail: tekkam212@gmail.com
Keywords Contrast-enhanced ultrasonography · Sonazoid · Laparoscopic colectomy $\cdot$ Live

\section{Introduction}

Colorectal cancer is the second leading cause of cancer deaths in Japan, as well as in Western countries, accounting for 34 and $26 \%$ of cancer in males and females, respectively [1]. Since the first reported laparoscopy-assisted colectomy (LAC) in 1991 [2], major advances have been achieved, particularly since the development of newer techniques and improved instruments, and the standardization of procedures. In 1992, LAC was first performed for early colorectal cancers in Japan [3], and many reports about laparoscopic surgery for advanced colorectal cancers have been published since [4-9].

Of the colorectal liver metastasis patients who undergo liver resection, $75 \%$ will develop recurrence, and the main site of recurrence is the liver $[1,10,11]$. In addition, $65-85 \%$ of all recurrences appear within the first 2 years because occult liver metastases are present at the time of liver resection [1, 10, 11]. Although contrast-enhanced computed tomography (CE-CT), magnetic resonance imaging (MRI) and positron emission tomography have been used to stage colorectal liver metastases, small or occult metastases are often not visualized. Conventional palpation of the liver and adequate observation of the abdominal cavity are also not achievable during laparoscopic surgery, and so cannot be used to determine if stage migration has occurred [12].

Intraoperative ultrasonography (IOUS) often reveals important information not detected on preoperative imaging, and these new findings can alter the surgical plan in up to $50 \%$ of patients $[13,14]$. Recently, several studies 
reported that contrast-enhanced IOUS (CE-IOUS) is more sensitive than conventional IOUS for identifying new lesions. It may thus influence surgical management $[1,11$, 15]. We hypothesized that the new, second-generation ultrasound microbubble agent, Sonazoid ${ }^{\circledR}$ (Daiichi Sankyo, Pharmaceutical, Tokyo, Japan), may improve the detection of occult liver metastases $[16,17]$. The aim of the present study was to assess the clinical value of Sonazoid-intraoperative laparoscopic ultrasonography (S-IOLUS) in patients with primary colorectal cancer. These results were historically compared with the data for conventional IOLUS.

\section{Methods}

A prospective database of patients treated within a single institution was studied retrospectively. From May 2005 to August 2008, 454 patients underwent colorectal resections for colorectal cancer at Iwate Medical University Hospital. The resections included 339 LACs $(75 \%)$ and 115 open colectomies (OCs) (25\%). Of the 454 patients, 148 consecutive patients with clinical stage II or III colorectal cancer, as determined by preoperative imaging, who were undergoing LACs were prospectively enrolled (Fig. 1). From May 2005 to December 2006, 77 of the 148 patients were examined without Sonazoid. Seventy-one of the 148 patients were examined with S-IOLUS after its approval in 2007 by Japan's health authorities. The present study was approved by the Iwate Medical University Institutional Review Board.

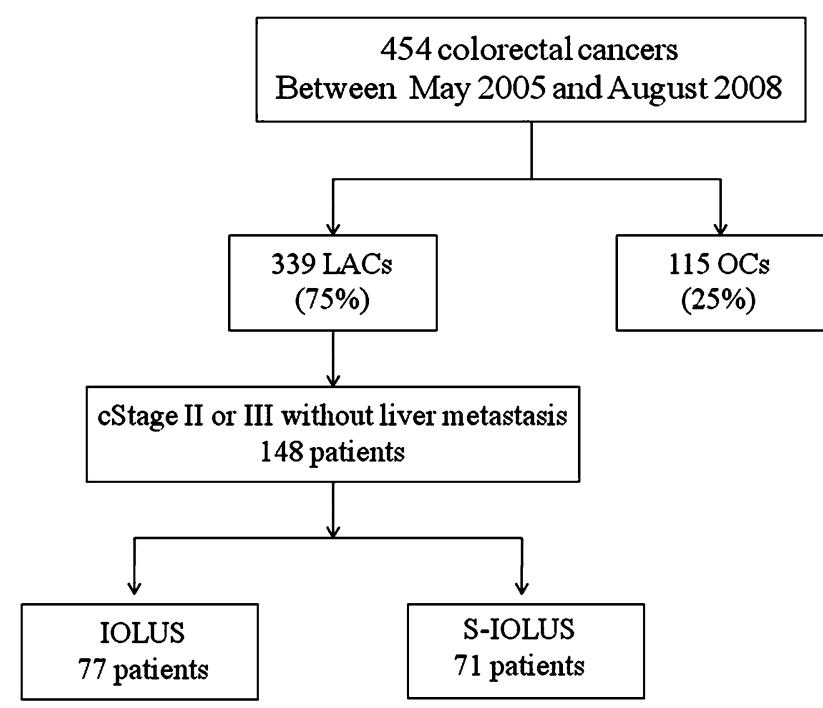

Fig. 1 A flowchart of the lesion detection. LAC laparoscopy-assisted colectomy, $O C$ open colectomy, IOLUS intraoperative laparoscopic ultrasonography, S-IOLUS Sonazoid-intraoperative laparoscopic ultrasonography
The clinical features are presented in Table 1. Of the 71 patients examined using S-IOLUS, 41 were male and 30 were female, and their median age was 72.5 years (range 46-83 years). The locations of the cancers included the colon (46) and the rectum (25). In this group, 60 patients (85\%) had T3 tumors and 28 patients (39\%) had lymph node metastasis. As a control group, the 77 patients who underwent LACs under conventional IOLUS between 2005 and April 2007 were used for comparison. The locations of their cancers included the colon (48) and the rectum (29). Of the 77 patients examined using IOLUS, 40 were male and 37 were female, and their median age was 70 years (range 45-88 years). In the control group, 57 patients (74\%) had T3 tumors and 38 patients (49\%) had lymph node metastasis.

All patients underwent CE-CT and/or MRI staging in a standardized manner within 4 weeks prior to surgery. CECT examinations were performed using a 16-detector scanner (Aquilion 16; Toshiba Medical Systems, Japan) with a slice thickness of $5 \mathrm{~mm}$ to screen the whole body. MRI was performed using a $1.5 \mathrm{~T}$ whole body MRI system (Signa Excite, Ver. 12, GE Medical Systems, Milwaukee, WI, USA). Preoperative images were reviewed by two different radiologists. CT films were available in the operating room during the laparoscopic procedure for an immediate comparative analysis after performing S-IOLUS. At this point, the final diagnosis of the presence or absence of liver metastasis was made by correcting the

Table 1 Patient characteristics

\begin{tabular}{llll}
\hline & S-IOLUS $(n=71)$ & IOLUS $(n=77)$ & $P$ value \\
\hline Age (years) & $68(46-83)$ & $69(45-88)$ & 0.61 \\
Sex, male/female & $41 / 30$ & $40 / 37$ & 0.48 \\
Tumor location & & & \\
Colon & 46 & 48 & \\
Rectum & 25 & 29 & 0.76 \\
Extent of the primary tumor & & \\
T2 & 11 & 20 & 0.12 \\
T3 & 60 & 57 & \\
Lymph node metastasis & & \\
Negative & 43 & 39 & 0.23 \\
Positive & 28 & 38 & \\
TNM stage & & 9 & 0.42 \\
I & 8 & 30 & \\
II & 35 & 38 & \\
III & 28 & & \\
\hline
\end{tabular}

The pathological staging was divided into subcategories according to American Joint Committee on Cancer

Data are expressed as medians (ranges)

S-IOLUS Sonazoid-intraoperative laparoscopic ultrasonography, IOLUS intraoperative laparoscopic ultrasonography 
diagnosis based on the pre- and perioperative images. All patients had followed-up examinations with ultrasonography and CE-CTs for at least 6 months postoperatively.

\section{IOLUS and S-IOLUS examinations}

The IOLUS and S-IOLUS examinations were performed using a Pro Focus UltraView device (BK Medical, Denmark) with a Type 8666 Laparoscopic Transducer. A bolus intravenous injection of Sonazoid $[(0.015 \mathrm{ml} / \mathrm{kg}$ body weight $(0.12 \mu \mathrm{L}$ microbubble/kg body weight as perflubutane microbubbles))] was performed via a peripheral venous line, followed by a $10 \mathrm{~mL}$ normal saline flush. Approximately, $10 \mathrm{~min}$ after the injection, the liver was scanned to observe a late Kupffer-phase image. The liver metastases were identified as filling defects that were clearer than those observed during the vascular phase. The main advantage of using Sonazoid over other agents is its capacity for obtaining continuous enhanced views in the Kupffer-phase 10-15 min after injection. The late Kupfferphase image lasted for $30 \mathrm{~min}$. The liver tumors without Kupffer cells were clearly revealed as low contrast perfusion defects compared with the normal liver parenchyma. Using these images, we examined the tumor morphology, surrounding daughter nodules and vascular infiltration. We also scanned for any tumor lesions that could not be identified by preoperative imaging.

\section{Statistical analysis}

Continuous data were expressed as the medians (ranges). To compare the examination groups, a Mann-Whitney $U$ test (two-tailed) was applied for the continuous data, and the $\chi^{2}$ test was applied for the categorical data. A value of $P<0.05$ was considered to be statistically significant.

\section{Results}

Preoperative CE-CT and/or MRI did not detect liver metastatic lesions in any of the 148 patients. All parenchymal segments were evaluated and all major vascular/biliary structures were identified by IOLUS or S-IOLUS in every patient. New lesions were identified by IOLUS or S-IOLUS in four patients (Table 2). No IOLUS-related complications occurred.

In the S-IOLUS group, two lesions in different patients $(2.8 \%)$ were identified and evaluated. Although IOLUS did not detect any new lesions, a small hypoechoic lesion that was $9 \mathrm{~mm}$ in diameter and located in segment 3 was newly detected by S-IOLUS in the Kupffer-phase view of one patient (Fig. 2). This lesion had increased in size 3 months after LAC, and laparoscopic left lateral sectionectomy was performed. The other patient had a lesion that was $7 \mathrm{~mm}$ in diameter and located in segment 8 . It was curatively resected by laparoscopic partial liver resection at the time of LAC. Both resected lesions were histopathologically confirmed to be metastatic tumors. None of the 71 patients showed liver metastases within 6 months after LAC (median follow-up 16 months; range 6-23 months).

In the IOLUS group, two lesions in different patients $(2.6 \%)$ were identified and evaluated. The first lesion was $11 \mathrm{~mm}$ in diameter and was located in segment 4. Laparoscopic segmentectomy (for segment 4 ) was performed on the patient 3 months after LAC. The lesion was histopathologically confirmed to be a metastatic tumor. The other lesion was $7 \mathrm{~mm}$ in diameter and was located in segment 6 . The patient developed multiple liver metastases 3 months after LAC and selected chemotherapy with a modified FOLFOX6 regimen $\left(400 \mathrm{mg} / \mathrm{m}^{2}\right.$ 5-fluorouracil, $85 \mathrm{mg} / \mathrm{m}^{2}$ oxaliplatin and $200 \mathrm{mg} / \mathrm{m}^{2}$ leucovorin on the first day of treatment, followed by $2,400 \mathrm{mg} / \mathrm{m}^{2}$ of 5 -fluorouracil on

Table 2 Clinical features of six patients with newly detected liver metastases

\begin{tabular}{llllll}
\hline Patient no. & Diagnostic procedure & Location $($ size, $\mathrm{mm})$ & Treatment (POMs) & Laparoscopic procedures or adjuvant therapy & TNM staging \\
\hline 1 & S-IOLUS & S3 $(9)$ & 3 & Left lateral sectionectomy & T3N1M1 \\
2 & S-IOLUS & S8 $(7)$ & Concomitant & S8 partial liver resection & T3N0M1 \\
3 & IOLUS & S4 $(11)$ & 3 & Segmentectomy 4 & T3N1M1 \\
4 & IOLUS & S6 (7) & 6 & mFOLFOX6 for MLM & T3N2M1 \\
5 & IOLUS & None & None & None & T3N0M0 \\
& P-CT & S2 (10) & 5 & Left lateral sectionectomy & T2N1M0 \\
& IOLUS & None & None & None & S4 partial liver resection + segmentectomy 5
\end{tabular}

S-IOLUS Sonazoid-intraoperative laparoscopic ultrasonography, IOLUS intraoperative laparoscopic ultrasonography, $P$-CT postoperative computed tomography, POMs postoperative months, FFOLFOX6 folinic acid/5-fluorouracil/oxaliplatin, $M L M$ multiple liver metastases 
Fig. 2 S-IOLUS and IOLUS images of a colon cancer metastasis at segment 3. a The metastatic lesion (arrows) was shown as a distinct hypoechoic mass during the late Kupfferphase. $\mathbf{b}$ The IOLUS view of the same lesion detected as an unclear slightly hypoechoic mass
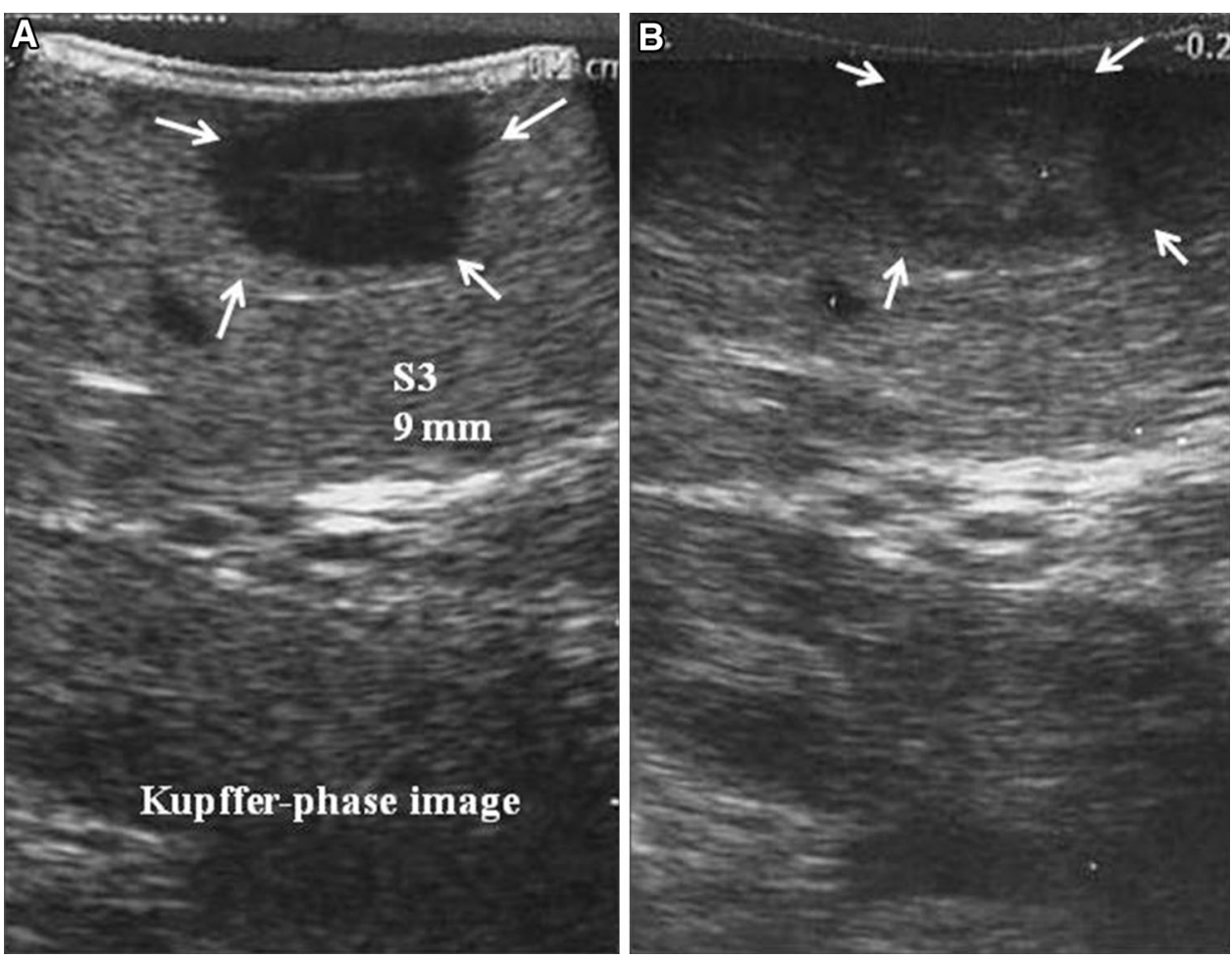

the first and second days of treatment, repeated every 2 weeks as a first-line treatment). Two (2.6\%) of the 77 patients developed liver metastases within 6 months after LAC (median follow-up 40 months; range 30-54 months). The new metastatic lesions could be detected by CE-CT, and the patients were operated on 6 months after LAC; one patient underwent a laparoscopic left lateral sectionectomy and the other patient underwent laparoscopic segmentectomy (segment 5) $+\mathrm{S} 4$ partial liver resection.

There were no significant differences between the two groups in the median length of the operation (S-IOLUS $158.5 \mathrm{~min}$ vs. IOLUS $169.0 \mathrm{~min}$ ).

\section{Discussion}

LAC was first described in 1991 [1], and the outcomes of LAC from members of the Clinical Outcomes of Surgical Therapy (COST) study group were first presented in 1996 and showed acceptable short-term results for the procedures $[18,19]$. Several studies have suggested that there are better long-term outcomes for LAC compared with OC [20-22]. A recent meta-analysis of 10 prospective randomized controlled trials demonstrated that there were trends toward lower recurrence rates and longer disease-specific survival rates with LAC [23]. In Japan, LAC had continued to gain widespread clinical acceptance since April 2002, when medical insurers extended the procedure for patients with advanced colorectal cancers. According to the Japanese
Society of Endoscopic Surgery, of the patients diagnosed with colorectal cancer in 2009 in Japan, 13,791 patients underwent LACs and 37,529 underwent OCs. From 2008 to 2009, the use of LACs increased from 33 to $37 \%$.

It is very important for liver metastasis to be accurately identified at primary colorectal surgery to select the best treatment strategy, and knowledge of the presence of liver metastasis can help to obtain an improved prognosis by complete resection. However, conventional palpation of the liver and sufficient observation of abdominal cavity are not achievable during laparoscopic surgery, and so, it is not possible to accurately determine whether stage migration has occurred [12]. Many studies have been reported that IOUS is an important tool that can be used to accurately stage colorectal liver metastases at the time of resection $[13,14]$. Recently, there has been increasing interest in the use of contrast agents during the IOLUS of the liver to improve the detection of liver metastases. In 2007, a new contrast medium, a microbubble agent, Sonazoid, was approved for use in Japan for the diagnosis of liver tumors, including hepatocellular carcinoma, cholangiocarcinoma and liver metastasis $[16,17]$. To the best of our knowledge, the application of S-IOLUS for colorectal cancers without liver metastases has not been reported.

Sonazoid is thicker than the previously used agents, and remains in the liver parenchyma for a long time after injection. Therefore, the reperfusion technique to determine intratumoral perfusion during the arterial phase or negative perfusion imaging during the Kupffer-phase can 
be conducted, because the enhanced lesions reflect sinusoidal cell function related to phagocytosis of the contrast agent by macrophages [24]. Several studies have shown that routine use of contrast-enhanced IOUS detects up to 19-25\% more occult metastatic lesions than preoperative CE-CT and IOUS, and that additional resection was often necessary during liver resections $[1,11]$. A phase III comparative study regarding the effectiveness of using Sonazoid to obtain the differential diagnosis of hepatic lesions reported that the diagnostic accuracy of unenhanced ultrasonography, contrast-enhanced $\mathrm{CT}$ and contrastenhanced ultrasonography was $68.4,80.5$ and $88.9 \%$, respectively. Among the 191 patients in the detection analysis in that study, the efficacy of contrast-enhanced ultrasonography for the detection of lesions was significantly higher than that of unenhanced ultrasonography and enhanced CT [25].

In the present study, occult metastatic lesions were detected by S-IOLUS in two of the 71 patients $(2.8 \%)$. In the IOLUS group, postoperative liver metastases were observed in two of the 77 patients (2.6\%) within 6 months after the LAC. However, in the S-IOLUS group, no liver metastases were observed within 6 months. Therefore, this new examination may help provide better surgical curability in comparison with conventional IOLUS.

Sonazoid has also been reported to be safe. The incidence of adverse events associated with Sonazoid was shown to be 25 of 397 patients (6\%) in a clinical study performed in Japan [11]. The main adverse events were diarrhea $(1 \%)$ and headache $(1 \%)$, but no anaphylactic shock was reported, unlike with CE-CT [11]. In the present study, no adverse events were observed; thus, this new examination can also be performed safely.

On the other hand, other microbubble contrast agents, such as Imavist ${ }^{\circledR}$ (Alliance, USA) and SonoVue ${ }^{\circledR}$ (Bracco $\mathrm{SpA}$, Milan, Italy), provide parenchyma-specific contrast by inducing a transient mechanical slowdown of microbubbles within the sinusoid, and these two contrast agents are only minimally phagocytosed by Kupffer cells, so they have a short period of visualization [1, 15]. Leen et al. [1] reported that CE-IOUS with SonoVue detected additional metastatic liver lesions during liver resections that were not identified with IOUS or CT/MRI in 13 of the 57 patients (23\%), and the smallest of the additional lesions measured $4 \mathrm{~mm}$. However, these two contrast agents cannot provide late Kupffer-phase images. In addition, the parenchymaspecific contrast images of these two microbubble agents can only be seen for 3-5 min after the injection, and repeated injections are needed during CE-IOUS to perform a whole liver examination. For these reasons, Sonazoid seems to be a superior microbubble contrast agent for CEIOLUS for precise liver examinations in patients vulnerable to occult liver metastases.
MRI is one of the most important tools used to detect liver tumors, but it is difficult and unrealistic to perform in all cases, including those where hepatic lesions are not suspected. Ultrasonography, especially S-IOLUS, has many advantages in terms of liver scanning, i.e. the safety of Sonazoid, particularly in patients with renal dysfunction or who are allergic to iodine. There is also no exposure to radiation during ultrasonography, unlike other imaging methods, and it is non-invasive and has no contraindications. Therefore, it is a useful technique to use as an alternative to intraoperative palpation for real-time observation of the liver. In addition, the surgeon can repeatedly scan the whole liver during surgery. This capability may help reduce the number of missed lesions.

Nevertheless, the effectiveness of scanning the entire liver with ultrasonography depends on the surgeons' experience levels and anatomical difficulties in the patient. If an inexperienced operator performs the liver scan, it is possible that occult metastatic lesions might be missed. In this study, scanning of the liver was performed by two surgeons who were certified by the Japan Society for Endoscopic Surgery and have been surgeons for more than 10 years. Therefore, it was unlikely that they would miss abnormal liver lesions. A clinical study comparing contrast $\mathrm{CT}$, MRI and contrast ultrasound examinations for the diagnosis of HCC found that these imaging techniques had a diagnostic accuracy of 74,86 and $72 \%$, respectively. However, no significant differences were observed between the groups in that study [26]. This suggests that the combination of CT and IOUS or MRI is sufficient to make a clinical diagnosis of liver metastasis.

With the increasing use of LACs for colorectal cancers, the value S-IOLUS should be assessed in a systematic fashion. It has already been shown to be an additional key staging component of the oncologic procedure. This precise staging may permit the earlier diagnosis of liver metastases, better selection of adjuvant therapy for patients and the application of valuable prognostic information.

\section{Conclusions}

S-IOLUS of the liver during primary resection of colorectal cancer is a useful tool for cancer staging and as a diagnostic modality. It can identify lesions that are not detectable by preoperative imaging studies, and may be considered for routine use during laparoscopic oncologic colorectal surgery. In addition to these benefits, S-IOLUS is also relatively safe to use.

Acknowledgments Tetsuya Itabashi and the co-authors have no conflicts of interest to declare. 
Open Access This article is distributed under the terms of the Creative Commons Attribution License which permits any use, distribution, and reproduction in any medium, provided the original author(s) and the source are credited.

\section{References}

1. Leen E, Ceccotti P, Moug S, et al. Potential value of contrastenhanced intraoperative ultrasonography during partial hepatectomy for metastases: an essential investigation before resection? Ann Surg. 2006;243:236-40.

2. Jacob M, Verdeja JC, Goldstein HS. Minimally invasive colon resection (laparoscopic colectomy). Surg Laparosc Endosc. 1991;1:144-50

3. Sekimoto M. Laparoscopic resection for colorectal cancer in Japan. Dis Colon Rectum. 2007;50:1708-14.

4. Hasegawa H, Kabeshima Y, Watanabe M, Yamamoto S, Kitajima M. Randomized controlled trial of laparoscopic versus open colectomy for advanced colorectal cancer. Surg Endosc. 2003;17:636-40.

5. Kitano S, Inomata M, Sato A, Yoshimura K, Moriya Y. Randomized controlled trial to evaluate laparoscopic surgery for colorectal cancer: Japan Clinical Oncology Group Study JCOG 0404. Jpn J Clin Oncol. 2005;35:475-7.

6. Naitoh T, Tsuchiya T, Honda H, Oikawa M, Saito Y, Hasegawa Y. Clinical outcome of the laparoscopic surgery for stage II and stage III colorectal cancer. Surg Endosc. 2008;22:950-4.

7. Miyajima N, Fukunaga M, Hasegawa H, Tanaka J, Okuda J, Watanabe M. Results of a multicenter study of 1,057 cases of rectal cancer treated by laparoscopic surgery. Surg Endosc. 2009;23:113-8.

8. Fukunaga Y, Higashino M, Tanimura S, Takemura M, Fujiwara Y, Osugi H. Laparoscopic surgery for stage IV colorectal cancer. Surg Endosc. 2010;24:1353-9.

9. Tan KY, Konishi F. Long-term results of laparoscopic colorectal cancer resection: current knowledge and what remains unclear. Surg Today. 2010;40:97-101.

10. Fong Y, Cohen AM, Fortner JG, Enker WE, Turnbull AD, Coit DG, et al. Liver resection for colorectal metastases. J Clin Oncol. 1997;15:938-46.

11. Nakano H, Ishida Y, Hatakeyama T, Sakuraba K, Hayashi M, Sakurai O, et al. Contrast-enhanced intraoperative ultrasonography equipped with late Kupffer-phase image obtained by Sonazoid $^{(\circledR)}$ in patients with colorectal liver metastases. World J Gastroenterol. 2008;14:3207-11.

12. Milsom JW, Jerby BL, Kessler H, Hale JC, Herts BR, O'Malley CM. Prospective, blinded comparison of laparoscopic ultrasonography vs. contrast-enhanced computerized tomography for liver assessment in patients undergoing colorectal carcinoma surgery. Dis Colon Rectum. 2000;43:44-9.

13. Jarnagin WR, Bach AM, Winston CB, Hann LE, Heffernan N, Loumeau $\mathrm{T}$, et al. What is the yield of intraoperative ultrasonography during partial hepatectomy for malignant disease? J Am Coll Surg. 2001;192:577-83.

14. Choti MA, Kaloma F, de Oliveira ML, Nour S, Garrett-Mayer ES, Sheth $\mathrm{S}$, et al. Patient variability in intraoperative ultrasonographic characterrictics of colorectal liver metastases. Arch Surg. 2008;143:29-34.

15. Tillaud H, Bruel JM, Valette PJ, Vilgrain V, Schmutz G, Oyen R, et al. Characterization of focal liver lesions with SonoVue ${ }^{\circledR}$ enhanced sonography: international multicenter-study in comparison to CT and MRI. World J Gastroenterol. 2009;15: 3748-56.

16. Uchiyama K, Ueno M, Ozawa S, Kiriyama S, Shigekawa Y, Yamaue H. Combined use of contrast-enhanced intraoperative ultrasonography and a fluorescence navigation system for identifying hepatic metastases. World J Surg. 2010;34:2953-9.

17. Nanashima A, Tobinaga $S$, Abo $T$, Kunizaki M, Takeshita $H$, Hidaka S, et al. Usefulness of Sonazoid-ultrasonography during hepatectomy in patients with liver tumors: a preliminary study. J Surg Oncol. 2011;103:152-7.

18. Nelson H, Weeks JC, Wieand HS. Prognosed phase III trial comparing laparoscopic-assisted colectomy versus open colectomy for colon cancer. J Natl Cancer Inst Monogr. 1995;19:51-6.

19. Fleshman JW, Nelson H, Peters WR, Kim HC, Larach S, Boorse $\mathrm{RR}$, et al. Early results of laparoscopic surgery for colorectal cancer: retrospective analysis of 372 patients treated by Clinical Outcomes of Surgical Therapy (COST) Study Group. Dis Colon Rectum 1996; 39 (sppl): S53-S58.

20. Clinical Outcomes of Surgical Therapy Study Group. A comparison of laparoscopically assisted and open colectomy for colon cancer. N Eng J Med. 2004;350:2050-9.

21. Jackson TD, Kaplan GG, Arena G, Page JH, Rogers SO Jr. Laparoscopic versus open resection for colorectal cancer: a metaanalysis of oncologic outcomes. J Am Coll Surg. 2007;204: 439-46.

22. Lacy AM, Delgado S, Castells A, Prins HA, Arroyo V, Ibarzabal A, et al. The long-term results of a randomized clinical trial of laparoscopy-assisted versus open surgery for colon cancer. Ann Surg. 2008;248:1-7.

23. Bilimoria KY, Bentrem DL, Nelson H, Stryker SJ, Stewart AK, Soper NJ, et al. Use and outcomes of laparoscopic-assisted colectomy for cancer in the United States. Arch Surg. 2008;143: 832-40.

24. Yanagisawa K, Moriyasu F, Miyahara T, Yuki M, Iijima H. Phagocytosis of ultrasound contrast agent microbubbles by $\mathrm{Ku}-$ pffer cells. Ultrasound Med Biol. 2007;33:318-25.

25. Moriyasu Fuminori, Itoh Kouichi. Efficacy of perflubutane microbubble-enhanced ultrasound in the characterization and detection of focal liver lesions: phase 3 multicenter clinical trial. Am J Roentgenol. 2009;193(1):86-95.

26. Alaboudy A, Inoue $\mathrm{T}$, Hatanaka $\mathrm{K}$, Chung $\mathrm{H}$, Hyodo $\mathrm{T}$, et al. Usefulness of combination of imaging modalities in the diagnosis of hepatocellular carcinoma using sonazoid-enhanced ultrasound, gadolinium diethylene-triamine-pentaacetic acid-enhanced magnetic resonance imaging, and contrast-enhanced computed tomography. Oncology. 2011;81:66-72. 